\title{
High-Dimensional Normalized Mutual Information for Image Registration Using Random Lines
}

\author{
A. Bardera, M. Feixas, I. Boada, and M. Sbert \\ Institut d'Informàtica i Aplicacions, Universitat de Girona, Spain \\ \{anton.bardera, miquel.feixas, imma.boada, mateu.sbert\}@udg.es
}

\begin{abstract}
Mutual information has been successfully used as an effective similarity measure for multimodal image registration. However, a drawback of the standard mutual information-based computation is that the joint histogram is only calculated from the correspondence between individual voxels in the two images. In this paper, the normalized mutual information measure is extended to consider the correspondence between voxel blocks in multimodal rigid registration. The ambiguity and highdimensionality that appears when dealing with the voxel neighborhood is solved using uniformly distributed random lines and reducing the number of bins of the images. Experimental results show a significant improvement with respect to the standard normalized mutual information.
\end{abstract}

\section{Introduction}

Multimodal image registration is an important component of medical image analysis used to match two images. It consists in finding the transformation that brings one image into the best possible spatial correspondence with the other one. A common method of solving the registration task is to treat it as a mathematical optimization problem, using a similarity measure to quantify the quality of the alignment of the two images for any given transformation.

Some information-theoretic measures, such as mutual information (MI) [1,2] and normalized mutual information (NMI) 3], have become a standard reference due to their accuracy and robustness. However, MI-based methods have a number of well-known drawbacks, such as grid [4 and interpolation effects [1,5]. Another limitation of these methods is that the computation of the joint histogram is calculated from the correspondence between individual voxels in the two images. In recent years, different approaches have considered a region-based correlation to compute image similarity $[6,7,8,9,10]$.

In this paper, we propose a new MI-based framework that uses structural information in an image. The NMI measure is extended to consider the correspondence between regions of voxels in multimodal rigid registration. The problems that appear when dealing with the voxel neighbourhood are tackled using uniformly distributed random lines. Experimental results analyze the behaviour of our approach when neighbour intensity values are considered. 
This paper is organized as follows. In Section 2 we survey background and related work, and in Section 3 we present our framework for image registration. Experimental results are given in Section 4

\section{Background and Related Work}

In this section we review some basic information-theoretic measures [11,12, the application of mutual information to image registration [1,2,13] and recent approaches which take into account spatial information.

\subsection{Information-Theoretic Measures}

Let $\mathcal{X}$ be a finite set, let $X$ be a random variable taking values $x$ in $\mathcal{X}$ with distribution $p(x)=\operatorname{Pr}[X=x]$. Likewise, let $Y$ be a random variable taking values $y$ in $\mathcal{Y}$. The Shannon entropy $H(X)$ of a random variable $X$ is defined by $H(X)=-\sum_{x \in \mathcal{X}} p(x) \log p(x)$ and measures the average uncertainty of random variable $X$. If the logarithms are taken in base 2, entropy is expressed in bits. The conditional entropy is defined by $H(X \mid Y)=-\sum_{x \in \mathcal{X}, y \in \mathcal{Y}} p(x, y) \log p(x \mid y)$, where $p(x, y)=\operatorname{Pr}[X=x, Y=y]$ is the joint probability and $p(x \mid y)=\operatorname{Pr}[X=$ $x \mid Y=y]$ is the conditional probability. The conditional entropy $H(X \mid Y)$ measures the average uncertainty associated with $X$ if we know the outcome of $Y$. The joint entropy $H(X, Y)$ is given by the Shannon entropy of the joint probability distribution. The mutual information between $X$ and $Y$ is defined by $I(X, Y)=H(X)-H(X \mid Y)=H(Y)-H(Y \mid X)$ and measures the shared information between $X$ and $Y$.

We review now the definition of block entropy and entropy rate. The notation used here is inspired by the work of Feldman and Crutchfield [12]. Given a chain $\ldots X_{-2} X_{-1} X_{0} X_{1} X_{2} \ldots$ of random variables $X_{i}$ taking values in $\mathcal{X}$, a block of $L$ consecutive random variables is denoted by $X^{L}=X_{1} \ldots X_{L}$. The probability that the particular $L$-block $x^{L}$ occurs is denoted by $p\left(x^{L}\right)$. The Shannon entropy of length- $L$ sequences or $L$-block entropy is defined by

$$
H\left(X^{L}\right)=-\sum_{x^{L} \in \mathcal{X}^{L}} p\left(x^{L}\right) \log p\left(x^{L}\right),
$$

where the sum runs over all possible $L$-blocks. The entropy rate is defined by

$$
h^{x}=\lim _{L \rightarrow \infty} \frac{H\left(X^{L}\right)}{L},
$$

and it can be written as $h^{x}=\lim _{L \rightarrow \infty} h^{x}(L)$, where $h^{x}(L)=H\left(X_{L} \mid X_{L-1} \ldots X_{1}\right)$ is the entropy of a symbol conditioned on a block of $L-1$ adjacent symbols. The entropy rate of a sequence measures the average amount of information per symbol $x$ and the optimal achievement for any possible compression algorithm [11]. 


\subsection{MI-Based Image Registration}

The crucial point of image registration is the choice of a metric. The registration between two images $X$ and $Y$ can be represented by an information channel $X \rightarrow$ $Y$, where its marginal and joint probability distributions are obtained by simple normalization of the corresponding intensity histograms of the overlap area of both images [1. The most successful automatic image registration methods are based on the MI maximization. This method, almost simultaneously introduced by Maes et al. [1] and Viola et al. 2, is based on the conjecture that the correct registration corresponds to the maximum MI between the overlap areas of the two images. Later, Studholme et al. 3] proposed a normalization of MI defined by

$$
N M I(X, Y)=\frac{I(X, Y)}{H(X, Y)}=1-\frac{H(X \mid Y)+H(Y \mid X)}{H(X, Y)},
$$

which is more robust than MI, due to its greater independence of the overlap area. The necessity of normalization is theoretically justified in [14. It should be noted that $1-N M I$ is a true distance (see [14,15]).

Standard MI-based measures ignore the spatial information contained in the images. Recent research has been focused on overcoming this problem. Rueckert et al. 6 propose to use a second-order MI to incorporate spatial information. The neighbourhood has been defined by the six nearest neighbours of each pixel. Pluim et al. [7 include spatial information by combining MI with a term based on the image gradient of the images to be registered. The gradient term seeks to align locations of high gradient magnitude and similar orientations of the gradients at these locations. Sabuncu and Ramadge $[8$ include spatial information in the MI-based approach by using spatial feature vectors obtained from the images and use a minimum spanning tree algorithm to estimate the conditional entropy in higher dimensions. Russakoff et al. 9] propose an MI extension which takes neighbourhood regions of corresponding pixels into account. They assume that the high-dimensional distribution is approximately normally distributed. Holden et al. [10] use the derivatives of gaussian scale space to provide structural information in the form of a feature vector for each voxel.

\section{Method}

In this section, we define the high-dimensional normalized mutual information and its computation using uniformly distributed random lines.

\subsection{High-Dimensional Normalized Mutual Information}

The $L$-dimensional normalized mutual information of an $L$-dimensional channel $X^{L} \rightarrow Y^{L}$ is defined by

$$
N M I\left(X^{L}, Y^{L}\right)=\frac{I\left(X^{L}, Y^{L}\right)}{H\left(X^{L}, Y^{L}\right)}=\frac{H\left(X^{L}\right)+H\left(Y^{L}\right)-H\left(X^{L}, Y^{L}\right)}{H\left(X^{L}, Y^{L}\right)},
$$

where the joint entropy $H\left(X^{L}, Y^{L}\right)$ is given by 


$$
H\left(X^{L}, Y^{L}\right)=-\sum_{x^{L} \in \mathcal{X}^{L}, y^{L} \in \mathcal{Y}^{L}} p\left(x^{L}, y^{L}\right) \log p\left(x^{L}, y^{L}\right),
$$

$I\left(X^{L}, Y^{L}\right)$ is the $L$-dimensional mutual information, and $p\left(x^{L}, y^{L}\right)$ is the joint probability of the $L$-dimensional channel. Now, $x^{L}$ stands for an $L$-block of intensity values in a given $3 \mathrm{D}$ region.

To compute the marginal and joint probabilities, how is $x^{L}$ selected?, i.e., how is the neighbourhood ambiguity solved? With respect to this problem, different templates have been proposed for two dimensions [12, but the generalization from 1-block approach to $L$-block approaches is a difficult problem.

Another aspect to be considered is the size of an $L$-block. As $L$ increases, $\frac{H\left(X^{L}\right)}{L}$ decreases, converging to the entropy rate $h^{x}$ of an image, which expresses its maximum compressibility or, equivalently, its irreducible randomness. In fact, the entropy of an image appears more random than it actually is, and the difference is given by $h^{x}(L)-h^{x}$ [12. Therefore, the higher the $L$ value, the better the approximation of $I\left(X^{L}, Y^{L}\right)$ to the true shared information. On the other hand, note that the number of elements of the joint histogram is given by $N^{2 L}$, where $N$ is the number of bins of an image.

From these limitations, how to compute $N M I\left(X^{L}, Y^{L}\right)$ for image registration? Rueckert et al. [6] propose a second-order MI using the six nearest neighbours of each voxel and reducing the number of bins to 16. One drawback of this approach is that not all directions are taken into account. In the next section, we tackle this problem using uniformly distributed random lines.

\subsection{Implementation Using Uniformly Distributed Random Lines}

Uniformly distributed random lines, also called global lines [16, can be utilized to compute the joint intensity histogram in 3D-image registration [17, which is the most demanding step in the similarity measure computation. The overlapping volume between two 3D-images is stochastically sampled using a uniform distribution of lines in the sense of integral geometry, i.e., invariant to translations and rotations [18. Points chosen on each line provide us with the intensity values to calculate the probability distributions.

A global line can be generated from the walls of a convex bounding box containing the data volume [19. This can be done taking a random point on the surface of the convex bounding box and a cosinus distributed random direction. This strategy is easily applicable to image registration since the reference image can be taken as the bounding box (Figure 11). The intensity values are captured from the lines at evenly spaced positions, taking an initial random offset different for each line. The random offset ranges from 0 to the step size. Although we skip with regular steps, the use of a random offset ensures the stochasticity of the process. This fact diminishes the interpolation artifacts (see [17]).

In order to implement the $N M I\left(X^{L}, Y^{L}\right)$ measure, we propose to take $L$ blocks on the global lines (Figure 11). Thus, the 3D-neighbourhood problem is reduced to a $1 \mathrm{D}$ problem. Now, the ambiguity about how to sample the neighbourhood disappears as the order in which we take the neighbours is well defined 

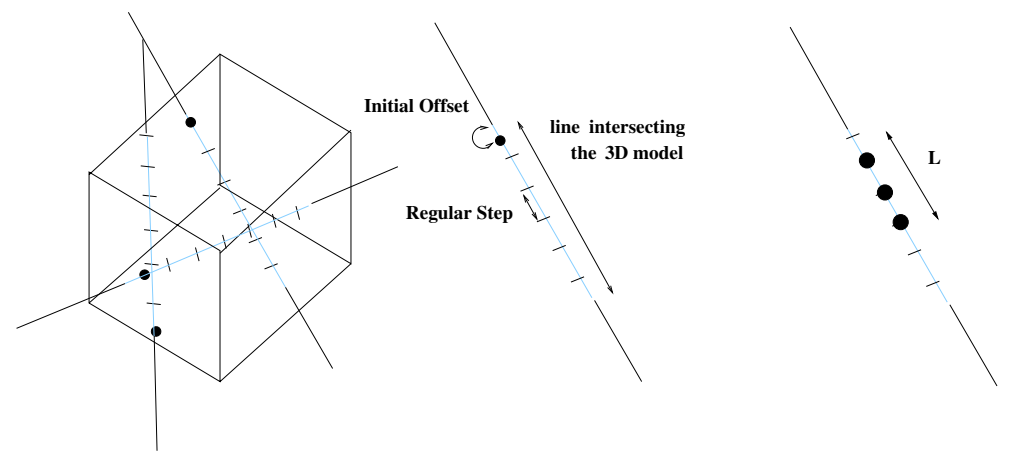

Fig. 1. Global lines are cast from the walls of the bounding box. Neighbour intensity values are taken in L-blocks.

on a global line. In spite of this, the dimensionality problem persists due to the fact that the computation of the joint histogram of the channel $X^{L} \rightarrow Y^{L}$ has an excessive cost if $L \geq 2$. Another problem is the sparsity of the resulting histograms since the number of bins of the joint histogram is $N^{2 L}$. A possible solution to these problems is to reduce the number of bins of the image, preserving a trade-off between level of binning and the accuracy of the entropy rate.

\section{Results}

In this section, two sets of experiments are carried out. First, we analyze the behaviour of $N M I\left(X^{L}, Y^{L}\right)$ when two neighbour intensity values are considered on random lines and, second, we show preliminary results taking 3-blocks. The clinical images and the standard transformations used for the tests are provided as part of the Retrospective Image Registration Evaluation project [20].

The first experiments use the ITK MI_Validation application [21] with a multiresolution optimization approach using the quaternion rigid transform gradient descent. In our implementation, three multiresolution steps, with 1500 iterations each one, are used. To reduce the computational cost, all the measures are computed using global lines, with a total number of 100000 sampling points and an step size of $2 \mathrm{~mm}$. The $N M I$ and $N M I\left(X^{2}, Y^{2}\right)$ have been computed, respectively, over a range of 256 and 16 bins per dimension. The results of CT-MR_T1 image registrations for 16 patients are shown in Table 1. Each entry in the table is the mean of the distances in $\mathrm{mm}$. between the evaluated registration method and the gold standard measured at different volumes of interest (VOI) of the patient. Note that in 14 out of 16 tested patients the proposed approach behaves better than the NMI method, and, in all the cases, the error of our method is lower than $2.5 \mathrm{~mm}$., which reveals its good performance. This good behaviour is also shown in Table 2 where the mean, the median and the maximum of the distances between VOIs have been computed from all patient results.

In Figure 2, we also present a preliminary evaluation of the proposed algorithm with blocks of three neighbours. Our method is now used to register the 
Table 1. The mean of the error in $m m$. for NMI and $N M I\left(X^{2}, Y^{2}\right)$, measured at different VOIs for each patient

\begin{tabular}{|c|r|c||c|r|c|}
\hline CT-MR_T1 & $N M I$ & $N M I\left(X^{2}, Y^{2}\right)$ & CT-MR_T1 & $N M I$ & $N M I\left(X^{2}, Y^{2}\right)$ \\
\hline pat_001 & 2.05 & 0.97 & pat_102 & 5.57 & 1.54 \\
pat_002 & 1.98 & 1.28 & pat_103 & 2.28 & 2.44 \\
pat_003 & 1.78 & 1.10 & pat_104 & 2.91 & 1.22 \\
pat_004 & 13.16 & 2.21 & pat_105 & 11.49 & 2.45 \\
pat_005 & 1.63 & 0.84 & pat_106 & 5.81 & 1.74 \\
pat_006 & 12.62 & 1.44 & pat_107 & 4.18 & 0.94 \\
pat_007 & 0.78 & 1.13 & pat_108 & 9.21 & 1.69 \\
pat_101 & 6.57 & 2.10 & pat_109 & 1.27 & 1.15 \\
\hline
\end{tabular}

Table 2. Mean, median and maximum of the distances in $\mathrm{mm}$. between VOIs from all patient results

\begin{tabular}{|c|c|c|c|}
\hline Method & Mean & Median & Maximum \\
\hline$N M I$ & 5.02 & 3.00 & 19.74 \\
\hline$N M I\left(X^{2}, Y^{2}\right)$ & 1.50 & 1.31 & 2.92 \\
\hline
\end{tabular}
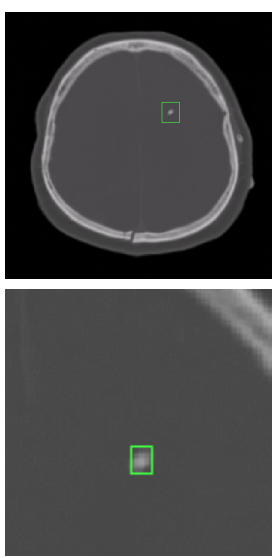

(a)CT with marker
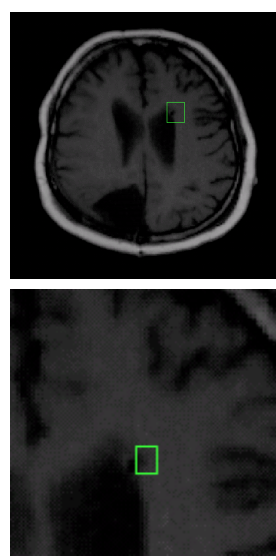

(b) $N M I(X, Y)$
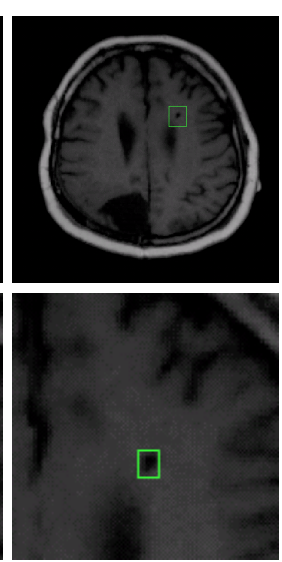

(c) $N M I\left(X^{2}, Y^{2}\right)$

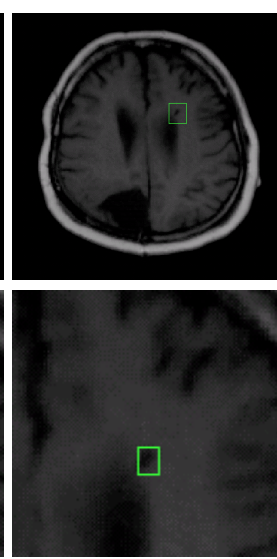

(d) $N M I\left(X^{3}, Y^{3}\right)$

Fig. 2. CT-MR registration results for different $L$ values

CT-MR_T1 pair of the patient 3 of the Vanderbilt database, considering different $\mathrm{L}$ values. To overcome the high-dimensionality of the joint histogram, the number of bins has been reduced to 16 bins and 8 bins for $L=2$ and $L=3$, respectively. In Figure 2, we show the original CT with a rectangular marker and the transformed MR obtained with $\mathrm{L}=1, \mathrm{~L}=2$ and $\mathrm{L}=3$ and with the marker in the same geometrical position as in the CT image. In the second row of Figure 2 we show a zoom of the first row images. In this test, the correctness of the registration for each case is determined by the degree of coincidence between the white dot in the center of the marker of the CT image and the black dot in the 
MR one. Observe that, for $N M I\left(X^{2}, Y^{2}\right)$ and $N M I\left(X^{3}, Y^{3}\right)$, the registration achieves more accurate results than the standard method NMI. This behaviour encourages us to explore the proposed measure with higher L values.

\section{Conclusions and Future Work}

In recent years, mutual information and its normalized versions have emerged as effective similarity measures for image registration. However, one significant drawback is that they ignore structural information in images. In this paper, we have presented the high-dimensional normalized mutual information which takes into account the dependence between image regions. The high dimensionality of the problem has been tackled using uniformly distributed random lines and reducing the number of image bins. Random lines permit us to solve the ambiguity in taking the neighbour intensity values of a voxel and also contributes to the reduction of interpolation artifacts. Experimental results show that an accurate and robust registration is achieved using only two neighbour intensity values in the normalized mutual information computation. In our future work we will analyze the behaviour of our proposal using three or four neighbours combined with a previous non-uniform quantization of the images.

\section{Acknowledgments}

The images used in our experiments were provided as part of the project, "Evaluation of Retrospective Image Registration", National Institutes of Health, Project Number 1 R01 NS33926-01, Principal Investigator Prof. J. Michael Fitzpatrick, Vanderbilt University, Nashville, TN. This project has been funded in part with grant numbers TIN2004-08065-C02-02, TIN2004-07451-C03-01 and 2001-SGR-00296.

\section{References}

1. Maes, F., Collignon, A., Vandermeulen, D., Marchal, G., Suetens, P.: Multimodality image registration by maximization of mutual information. IEEE Transactions on Medical Imaging 16(2) (1997) 187-198

2. Viola, P.A.: Alignment by Maximization of Mutual Information. PhD thesis, Massachusetts Institute of Technology, Massachusetts (MA), USA (1995)

3. Studholme, C.: Measures of 3D Medical Image Alignment. PhD thesis, University of London, London, UK (1997)

4. Unser, M., Thévenaz, P.: Stochastic sampling for computing the mutual information of two images. In: Proceedings of the 5th International Workshop on Sampling Theory and Applications (SampTA'03). (2003) 102-109 Held in Strobl, Austria.

5. Tsao, J.: Interpolation artifacts in multimodal image registration based on maximization of mutual information. IEEE Transactions on Medical Imaging 22 (2003) $854-864$ 
6. Rueckert, D., Clarkson, M.J., Hill, D.L.G., Hawkes, D.J.: Non-rigid registration using higher-order mutual information. In: Proc. SPIE Medical Imaging 2000: Image Processing. (2000) 438-447 San Diego.

7. Pluim, J.P., Maintz, J., Viergever, M.A.: Image registration by maximization of combined mutual information and gradient information. IEEE Transactions on Medical Imaging 19(8) (2000) 809-814

8. Sabuncu, M.R., Ramadge, P.J.: Spatial information in entropy-based image registration. In: Proceedings of 2nd Workshop in Biomedical Image Registration (WBIR'03), LNCS 2717. (2003) 132-141 Held in Philadelphia, USA.

9. Russakoff, D.B., Tomasi, C., Rohlfing, T., C. R. Maurer, J.: Image similarity using mutual information of regions. (In: Proceedings of the 8th European Conference on Computer Vision (ECCV), LNCS 3023) 596-607

10. Holden, M., Griffin, L.D., Hill, D.L.G.: Multi-dimensional mutual information image similarity metrics based on derivatives of linear scale space. In Lovell, B.C., Maeder, A.J., eds.: Proceedings of the APRS Workshop on Digital Image Computing. (2005) 55-60

11. Cover, T.M., Thomas, J.A.: Elements of Information Theory. Wiley Series in Telecommunications (1991)

12. Feldman, D.P., Crutchfield, J.P.: Structural information in two-dimensional patterns: Entropy convergence and excess entropy. (2002)

13. Pluim, J.P., Maintz, J., Viergever, M.: Mutual-information-based registration of medical images: a survey. IEEE Transactions on Medical Imaging 22 (2003) 9861004

14. Li, M., Chen, X., Li, X., Ma, B., Vitányi, P.: The similarity metric. IEEE Transactions on Information Theory 50(12) (2004) 3250-3264

15. Kraskov, A., Stögbauer, H., Andrzejak, R.G., Grassberger, P.: Hierarchical clustering based on mutual information. (2003)

16. Sbert, M.: An integral geometry based method for fast form-factor computation. Computer Graphics Forum (Proceedings of Eurographics'93) 12(3) (1993) 409-420 Held in Barcelona, Spain.

17. Bardera, A., Feixas, M., Boada, I., Sbert, M.: Medical image registration based on random line sampling. In: IEEE International Conference on Image Processing (ICIP'05), Proceedings, Genova, Italy (2005)

18. Santaló, L.A.: Integral Geometry and Geometric Probability. Addison-Wesley, Reading (MA), USA (1976)

19. Castro, F., Martínez, R., Sbert, M.: Quasi-Monte Carlo and extended first shot improvements to the multi-path method. In: Proceedings of Spring Conference on Computer Graphics'98. (1998) 91-102 Held in Budmerice, Slovak Republic.

20. National Institutes of Health: Retrospective Image Registration Evaluation. Vanderbilt University, Nashville (TN), USA. (2003) Project Number 8R01EB00212403, Principal Investigator J. Michael Fitzpatrick.

21. ITK Insight Toolkit, http://www.itk.org. 